\title{
Uretrolisis transvaginal tras cirugía correctora de la incontinencia urinaria de esfuerzo
}

\author{
P.C.R. Palma, M. Dambros, C.L.Z. Riccetto, M. Thiel, N. Rodrigues Netto Jr. \\ Servicio de Uroginecología, Universidade Estadual de Campinas-UNICAMP, Campinas, Brasil.
}

Actas Urol Esp 2005; 29 (2): 207-211

\section{RESUMEN \\ URETROLISIS TRANSVAGINAL TRAS CIRUGIA CORRECTORA DE LA INCONTINENCIA URINARIA DE ESFUERZO}

Objetivos: Analizar los resultados de la uretrolisis transvaginal como medida terapéutica para la disfunción miccional, en pacientes sometidas previamente a cirugia anti-incontinencia.

Materiales y Métodos: Fue realizado un estudio retrospectivo, en 20 pacientes quienes fueron sometidas a uretrolisis por obstrucción uretral secundaria a una cirugia anti-incontinencia. Pre-operatoriamente fueron realizados historia, examen físico y estudio urodinámico. La edad media de las pacientes fue 48 años. El tiempo de evolución entre la cirugía y la uretrolisis, varió de 3 meses a 8 años. Cuatro pacientes presentaron retención urinaria y las demás referían sintomas irritativos. Las cirugías previas incluían: sling pubo vaginal en 11 pacientes, uretropexia retropúbica en 3 y suspensión del cuello vesical con aguja en 6 . El seguimiento posterior a la realización de la uretrolisis fue de 14 meses. La evaluación urodinámica demostró un flujo urinario promedio de $9,9 \mathrm{ml} / \mathrm{s}$ y presión detrusora media en el flujo máximo de $48 \mathrm{cmH} 2 \mathrm{O}$.

Resultados: Aproximadamente $70 \%$ de las pacientes presentaron mejoría subjetiva total de los síntomas. Dos pacientes presentaron falla del método y fueron sometidas a nueva uretrolisis con interposición de flap de Martius. No hubo correlación entre los parámetros clínicos y urodinámicos encontrados en el pre operatorio y los resultados posteriores a la uretrolisis.

Conclusión: Tratamos de demostrar que la uretrolisis posterior a cirugía anti-incontinencia es un procedimiento efectivo, mínimamente invasivo que permite resultados satisfactorios para el tratamiento de la disfunción miccional que no se resuelve espontáneamente.

Palabras clave: Obstrucción uretral. Incontinencia urinaria. Uretrolisis.

\section{ABSTRACT}

TRANSVAGINAL URETHROLYSIS FOR URETHRAL OBSTRUCTION AFTER ANTI-INCONTINCE SURGERY

Purpose: To analyse the success of transvaginal urethrolysis in resolving voiding dysfunction in patients

following an anti-incontinence procedure.

Materials and Methods: A retrospective chart review was performed on 20 patients who had undergone transvaginal urethrolysis for urethral obstruction following an anti-incontinence procedure. Preoperatively, a history was taken, pelvic examination and urodynamic were done. Mean patient age was 48 years, and the median time between the anti-incontinence procedure and the urethrolysis was nine months. Four patients had urinary retention and had irritative voiding symptoms. Previous surgery included pubovaginal sling in eleven patients, retropubic urethropexy in three and bladder neck suspension in six cases. Mean length of follow up after urethrolysis was 14 months. The urodynamic study demonstrated voiding flow rate of $9,9 \mathrm{ml} / \mathrm{s}$ and detrusor pressures at maximum flow of $48 \mathrm{cmH} 2 \mathrm{O}$.

Results: Of the 20 patients 14 (70\%) had relief of symptoms after a single urethrolysis, while two patients underwent a second transvaginal urethrolysis, with placement of a Martius flap between the urethra and the symphysis. There was no correlation between preoperative parameters examined and the outcome from urethrolysis.

Conclusion: Our data support transvaginal urethrolysis for the treatment of urethral obstruction after anti-incontinence surgery. It is effective and minimally invasive technique with good results that should be considered if voiding dysfunction does not resolve spontaneously.

Keywords: Urethral obstruction. Urinary incontinence. Urethrolysis. 
$\mathrm{L}^{2}$ a corrección quirúrgica de la incontinencia urinaria de esfuerzo es un procedimiento urológico común que puede ser hecho de varias maneras, incluimos cistouretropexia retropúbica y procedimientos de sling pubo vaginal, también con la finalidad de reposicionar y dar soporte al cuello vesical y la uretra media ${ }^{1,2}$. La complicación potencial de todos estos procedimientos que quirúrgicamente alteran la anatomía, es la obstrucción urinaria iatrogénica llevando a disfunción miccional. Esta puede llevar a sintomas obstructivos con retención urinaria parcial o total, infección urinaria recurrente o sintomas irritativos severos, como frecuencia, urgencia y urge-incontinencia ${ }^{3}$. Los síntomas de urge-incontinencia y urgencia son más frecuentes que la retención franca ${ }^{4}$. La incidencia de obstrucción después de los procedimientos antiincontinencia no es clara, se estima entre 2,5 y $24 \%$ para las diferentes técnicas ${ }^{5}$.

Fueron demostrados índices de obstrucción entre un 5 a 20\% con la técnica de MarshallMarchetti-Krantz ${ }^{6}$, 5 a 7\% después de suspensión con aguja ${ }^{7}$ y 4 a $10 \%$ posterior a sling pubo vaginal $^{8}$.

Diferentes técnicas para uretrolisis transvaginal han sido descritas en la literatura con buenos resultados 9 . Presentamos nuestra experiencia con uretrolisis transvaginal en 20 pacientes con sintomas de obstrucción urinaria posterior a la realización de cirugía anti-incontinencia.

\section{MATERIALES Y MÉTODOS}

Fue realizado un estudio retrospectivo en 20 pacientes quienes fueron sometidas a uretrolisis transvaginal por obstrucción uretral posterior a un procedimiento anti-incontinencia entre 1994 y 2002. La edad media fue 48 años (rango 3168), el tiempo promedio entre la primera cirugía y la uretrolisis fue de 9 meses (variando entre 3 meses y 8 años). El seguimiento posterior a la uretrolisis fue 14 meses. La evaluación pre-operatoria incluyó historia clínica, examen físico y evaluación urodinámica.

Aproximadamente $72 \%$ de las pacientes referían sintomas irritativos posterior a la cirugía anti-incontinencia (disuria, urgencia, nicturia) y $20 \%$ presentaron retención aguda de orina. Los procedimientos anti-incontinencia realizados fueron: sling pubo vaginal 55\%, suspensión del cuello vesical con aguja (técnica Sholomo Raz) $30 \%$ y suspensión retro púbica (MarshallMarchetti-Krantz) en 15\%. Todas las pacientes referían por lo menos dos embarazos.

El examen físico demostró presencia de cistocele en $10 \%$ de las pacientes. Aproximadamente $50 \%$ presentaban examen vaginal sugestivo de hiper suspensión uretral. La uretra se encontraba fija a los planos vecinos.

La evaluación urodinámica demostró flujo urinario máximo variando entre 3,9 a $10,2 \mathrm{ml} / \mathrm{s}$ en una media de $9,9 \mathrm{ml} / \mathrm{s}$. La presión del detrusor en el flujo máximo varió de 36 a 84 cmH2O con una media de $48 \mathrm{~cm} H 2 \mathrm{O}$. Siete pacientes (35\%) presentaban presión detrusora menor $40 \mathrm{cmH} 2 \mathrm{O}$. Tres (15\%) presentaban volumen residual mayor de $200 \mathrm{ml}$ y siete (35\%) manifestaron contracciones no inhibidas del detrusor. No hubo pérdida de orina durante la fase de llenado ni con maniobras para provocarlas en ninguna paciente.

El criterio para indicar la uretrolisis fue seguimiento de los sintomas miccionales posterior a la cirugía anti-incontinencia con duración superior a los tres meses. El diagnóstico de areflexia del detrusor fue descartado con el estudio urodinámico.

Descripción de la técnica quirúrgica: El procedimiento fue realizado con raquianestesia y el paciente en posición de litotomía. Posterior a la realización de asepsia y antisepsia del campo operatorio, se realizó una incisión en U invertido en la pared vaginal $1 \mathrm{~cm}$ por debajo del meato uretral, extendiéndose en dirección al cuello uterino. Fue colocada una sonda de Foley número $16 \mathrm{~F}$ transuretral y dejada durante todo el procedimiento. Del epitelio vaginal, fue disecada la uretra. Dicha disección fue realizada bilateralmente, separando la uretra del tejido fibrótico que la rodeaba y entrando en el espacio retropúbico con tijera. La uretra fue circundada, con una pinza que la envolvió a través del espacio hecho por la disección. Con esta maniobra se comprobó la movilización completa de la uretra.

El catéter de Foley fue dejado por 24 horas.

El seguimiento de los pacientes fue basado en la evolución clínica, examen pélvico y medición del volumen residual realizado cada 3 meses hasta que la paciente no presentase más ninguno síntoma miccional. 
Para la clasificación de los síntomas urinarios posterior a la uretrolisis se realizó una cuantificación subjetiva y se distribuyeron en tres grupos: mejora total (sin molestias miccionales) mejora parcial (disminución de aproximadamente 50\% de las molestias miccionales) falla del procedimiento (persistencia de los sintomas miccionales)

Para análisis estadístico de la relación entre la técnica quirúrgica anti-incontinencia, la presencia de síntomas obstructivos y de los resultados obtenidos con la uretrolisis, fue utilizado el test de Fischer. Para análisis de el tiempo transcurrido entre la obstrucción y de los resultados obtenidos con la cirugía fue utilizada una regresión logística con un alfa de 5\%.

\section{RESULTADOS}

Fue identificada mejora total de los sintomas en $70 \%$ de las pacientes, posterior al seguimiento medio de 14 meses. Dos pacientes (10\%) presentaron persistencia de retención urinaria después de 2 meses de post operatorio por lo que fueron sometidas a nueva uretrolisis con interposición de flap de Martius (entre la uretra y la sinfisis púbica) (Figs. 1, 2 y 3) con mejora total de los síntomas posterior al procedimiento. Las demás pacientes presentaban mejoría parcial.

El examen pélvico no demostró alteraciones sugestivas de hiper-suspensión uretral y la cuan-

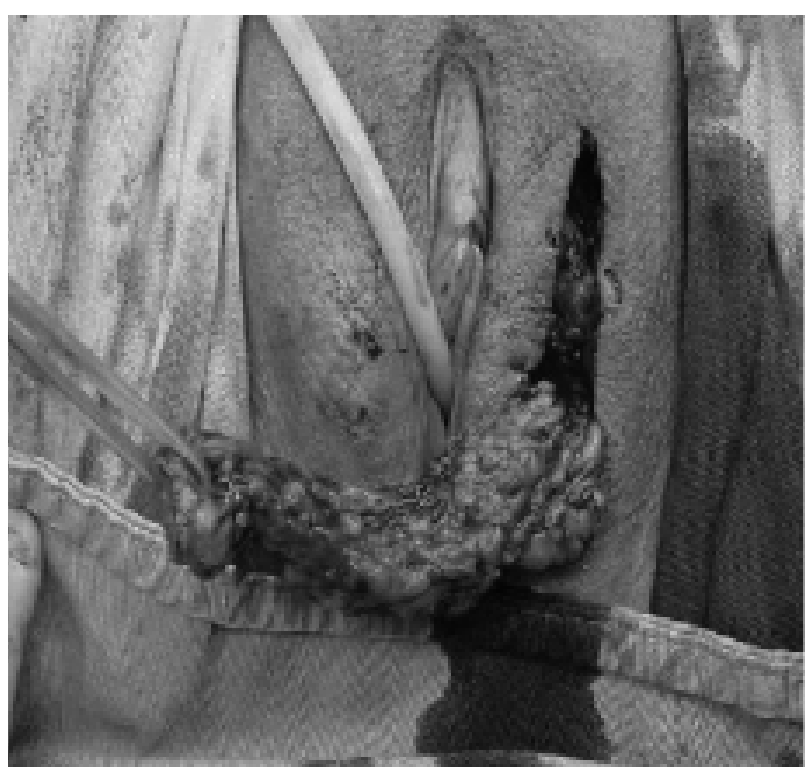

FIGURA 1. Flap pediculado del gran labio vaginal.

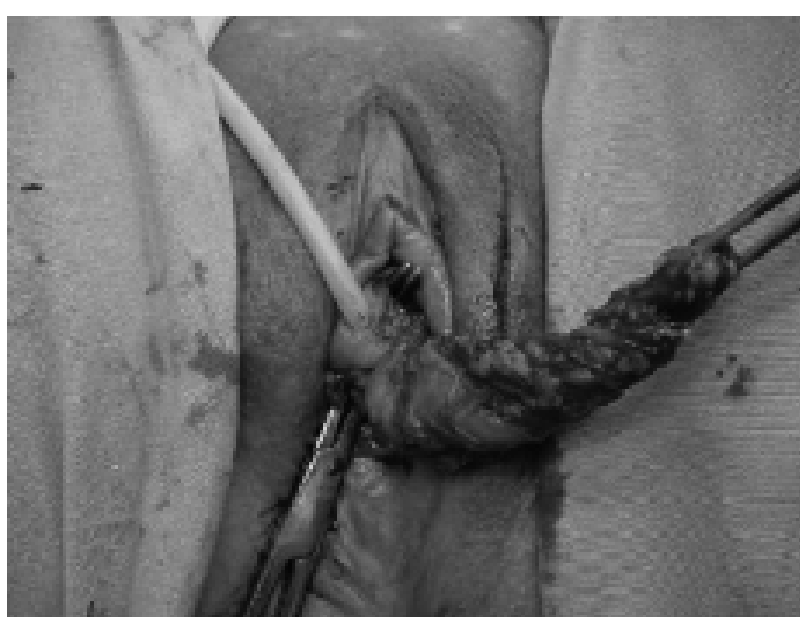

FIGURA 2. Liberación de la parte posterior de la uretra.

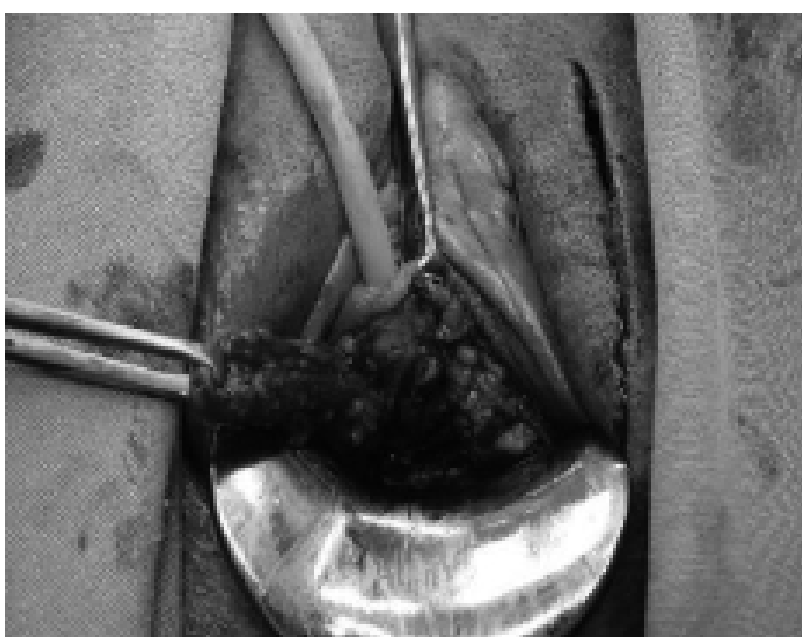

FIGURA 3. Pasaje del flap por detrás de la uretra.

tificación del residuo post miccional se encontró alterado en una paciente (mayor de $100 \mathrm{ml}$ ), ameritando utilizar maniobra de Valsalva para vaciar adecuadamente la vejiga.

No hubo diferencia en cuanto a la respuesta terapéutica cuando comparamos el tiempo entre la cirugía anti-incontinencia, la uretrolisis y la técnica quirúrgica previa (Tabla 1).

$\mathrm{Al}$ analizar los estudios urodinámicos pre-operatorios para determinar si había algún factor indicativo que modificase la evolución terapéutica, detectamos la misma respuesta quirúrgica en aquellas pacientes con anomalías usualmente con obstrucción, como inestabilidad del detrusor, presión del detrusor elevada, residuo vesical aumentado y en los pacientes sin alteraciones.

Ninguna paciente, clasificaba con mejora total de los síntomas, no se presentó incontinencia 
Tabla 1

Resultado clínico de la uretrolisis transvaginal en relación al tipo de cirugía anti-incontinencia y el tiempo transcurrido entre ésta y la uretrolisis

Grupo

uspensión con aguja

(número de pacientes $=6$ )

Uretropexia retro púbica

(número de pacientes $=3$ )

Sling pubo vaginal

(número de pacientes $=11$ )
Tiempo entre la cirugía anti-incontinencia y uretrolisis (promedio en meses)

7

12

9
Resultados

mejora total

(subjetiva)

$69 \%$

$70 \%$

$71 \%$

son volumen residual post-miccional elevado y examen físico sugestivo de exceso de suspensión de la uretra ${ }^{9}$. En nuestro estudio, ninguno de los parámetros clínicos y urodinámicos examinados, como técnica quirúrgica para incontinencia, tiempo entre esta cirugía y la uretrolisis, flujo miccional máximo y presión detrusora, demostraron correlación en los resultados obtenidos en la uretrolisis. Nuestros datos no nos

urinaria ni recurrencia de los síntomas después de la uretrolisis transvaginal, durante el seguimiento medio de 14 meses.

\section{DISCUSIÓN}

La obstrucción uretral posterior a un procedimiento para incontinencia urinaria se puede presentar entre un 4 y un $20 \%$ de los $\operatorname{casos}^{6}$. Inicialmente los pacientes son tratados con cateterismo intermitente, dilatación uretral y medicación anticolinérgica. Si los síntomas persisten por más de 3 meses y son refractarios a las conductas conservadoras, la resolución quirúrgica se hace necesaria. Varios factores pueden predisponer a los pacientes a presentar dificultad miccional posterior a una cirugía para la corrección de pérdida urinaria, como el gran volumen residual y la vejiga de gran capacidad ${ }^{9}$.

Los procedimientos realizados para la corrección de la incontinencia pueden llevar a obstrucción por varios factores. La localización de las suturas medialmente, puede cerrar la uretra, causar desvío y cicatrices periuretrales. Suturas colocadas distalmente, causan angulación exagerada pudiendo llevar a obstrucción e incontinencia, ya que el cuello vesical y la uretra proximal no fueron adecuadamente elevados. Otras causas incluyen fibrosis periuretral, causadas por suturas realizadas a través de la luz uretral con posterior extravasación de orina y fibrosis ${ }^{10}$.

Criterios absolutos para el diagnóstico de obstrucción en mujeres no fueron descritos y la causa exacta de los síntomas de urge-incontinencia en estas pacientes no fue establecida. La gran mayoría de los casos, los hallazgos usuales en las quejas de urgencia y urge-incontinencia permiten proporcionar una observación mas cuidadosa de algún parámetro como forma de predecir la respuesta en la uretrolisis.

Algunos estudios recomiendan la evaluación de la disminución del flujo urinario en pacientes con sospecha de obstrucción uretral, sin embargo la tasa de flujo no fue demostrada en el método perfectamente y no fueron descritos los parámetros adecuados para su evaluación. Farrar usó solamente el flujo máximo para el diagnóstico de obstrucción por que el considera que un flujo bajo con la presencia de presión detrusora normal o elevada puede ser indicativo de obstrucción, definida como flujo máximo menor de $15 \mathrm{ml} / \mathrm{s}$ con un volumen urinario mayor de $200 \mathrm{ml}^{11}$. Estudios como análisis de flujo-presión, presión detrusora en el flujo máximo y medición de la resistencia uretral han sido usados en los variados grados de éxito para el diagnóstico de la obstrucción ${ }^{12}$. Massey y Abrams propusieron que dos o más de los siguientes parámetros, deberían estar presentes para el diagnóstico de la patología: flujo máximo menor de $12 \mathrm{ml} / \mathrm{s}$, presión del detrusor en el flujo máximo mayor de $50 \mathrm{cmH} 2 \mathrm{O}$, resistencia uretral mayor de 0,2 con volumen residual urinario significativo, la presencia de presión detrusora elevada o resistencia elevada ${ }^{13}$. La evaluación urodinámica y simultáneamente la medición de la presión intravesical e intrauretral, promueve información sobre la actividad del esfinter uretral en visualización del área de la obstrucción durante la contracción vesical ${ }^{9}$.

En nuestro estudio los resultados fueron satisfactorios en aproximadamente $85 \%$ de las pacientes. Utilizamos la técnica transvaginal y no realizamos, rutinariamente, un procedimiento para incontinencia, a menos que la evaluación 
urodinámica demostrara pérdida de orina. En el grupo estudiado no hubo ninguna paciente en diagnóstico de incontinencia urinaria. Nuestros resultados son semejantes a otros estudios que reportan mejora total o parcial entre un 80 y $90 \%$ del grupo estudiado. McGuire, usando abordaje transvaginal, sin un procedimiento para incontinencia asociado, encontró micción normal en $77 \%$ de las pacientes posterior a la cirugía ${ }^{14}$. En un estudio posterior, involucrando un mayor número de pacientes y realizando sling pubo vaginal para los casos con pérdida de orina, se alcanzaron resultados positivos en alrededor del $85 \%$ de los casos 9 .

En los casos que persistió la retención urinaria, las pacientes fueron sometidas a nueva uretrolisis, con colocación concomitante de flap de Martius, con resultados satisfactorios. Carr obtuvo buena respuesta clínica en la interposición de flap de Martius entre la uretra y la sínfisis, posterior a uretrolisis sin éxito ${ }^{15}$.

El diagnóstico de obstrucción urinaria posterior a cirugía para incontinencia puede dificultarse, debido a la ausencia de parámetros claramente definidos para esta patología.

En cuanto, a la persistencia de síntomas miccionales que sugieran la presencia de obstrucción, a la detección de patrones urodinámicos que demuestren dificultad de vaciamiento vesical, y la ausencia de areflexia detrusora la uretrolisis es el método efectivo y seguro, presentando buenos resultados a pesar de la dificultad presentada en la definición de los hallados urodinámicos y el diagnóstico de obstrucción, entendemos la necesidad del examen, principalmente en pacientes que no presentaron retención total y un residuo miccional elevado, posterior a cirugía para incontinencia.

\section{CONCLUSIÓN}

Nuestro estudio demostró que la uretrolisis es un procedimiento efectivo para el tratamiento de la retención urinaria y la disfunción miccional que se presenta posterior a un procedimiento anti-incontinencia. Siendo que nuestras pacientes no presentaban pérdida urinaria solo fue evaluada la efectividad de la uretrolisis aisladamente sin un procedimiento anti-incontinencia en el mismo acto operatorio, cuando la queja de incontinencia se presentaba presente.

\section{REFERENCIAS}

1. Marshall VF, Marchetti AA and Krantz KE. The correction of stress incontinence by simple vesicourethral suspension. Surg Gynec \& Obst 1949;88:509-515.

2. Stamey TA. Endoscopic suspension of the vesical neck for urinary incontinence in females. Report on 203 consecutive patients. Ann Surg 1980;192:465-471.

3. Nitti VW, Raz S. Obstruction following anti-incontinence procedures: diagnosis and treatment with transvaginal urethrolyis. J Urol 1994;152:93-98.

4. Weinberg MW, Ostergard DR. Long-term clinical and urodynamic evaluation of the polytetrafluorethylene suburethral sling for treatment of genuine stress incontinence. Obst Gynec 1995;86:92-98.

5. Juma S, Sdrales L. Etiology of urinary retention after bladder neck suspension. J Urol 1993; part 2, 149:401 A, abstract 752 .

6. Zimmern PE , Hadley HR, Leach GE, Raz S. Female urethral obstruction after Marshall-Marchetti-Krantz operation. J Urol 1987;138:517-520.

7. Holschneider CH, Solh S, Lebhertz TB and Montz FJ. The modified Pereyra procedure in recurrent stress urinary incontinence: a 15 year review. Obst Gynec 1994;83:573-578.

8. Horbach NS. Suburethral sling procedures. In: Urogynecology \& Urodynamics Theory and Practice, $3^{\text {rd }}$ ed. Edited by DR Ostergard and AE Bent. Baltimore: Williams \& Wilkins 1991;413-421.

9. Cross CA, Cespedes D, English SF, McGuire E. Transvaginal urethrolysis for urethral obstruction after anti-incontinence surgery. J Urol 1988;159:1199-1201.

10. Bass JS, Leach GE. Bladder outlet obstruction in women. Probl Urol 1991;5:141-146.

11. Farrar DJ, Osborne JL, Stephenson TP, Whiteside CG, Weir J, Berry J, Milroy EJG, Turner Warwick R. A urodynamic view of bladder outflow obstruction in the female: factors influencing the results of treatment. Brit J Urol 1976;47:815-819.

12. Webster G, Kreder K. Voiding dysfunction following cystourethropexy: its evaluation and management. J Urol 1990;144:670-673.

13. Massey JÁ, Abrams PH. Obstructed voiding in the female. Brit J Urol 1988;61:36-99.

14. McGuire EJ, Letson W, Wang S. Transvaginal urethrolysis after obstructive urethral suspension procedures. J Urol 1989;142:1037-1038.

15. Carr LK, Webster GD. Voiding dysfunction following incontinence surgery: diagnosis and treatment with retropubic or vaginal urethrolysis. J Urol 1997;157:821-823.

Dra. M. Dambros

Rua Tiradentes, 426/71

13023-191 Campinas, SP, Brasil

(Trabajo recibido el 26 octubre 2004) 